\title{
"Go Away": Participant Objections to Being Studied and the Ethics of Chatroom Research
}

\author{
James M. Hudson and Amy Bruckman \\ College of Computing, Georgia Institute of Technology, Atlanta, Georgia, USA
}

In this article we present an empirical study aimed at better understanding the potential for harm when conducting research in chatrooms. For this study, we entered IRC chatrooms on the ICQ network and posted one of three messages to tell participants that we were recording them: a recording message, an opt-in message, or an opt-out message. In the fourth condition, we entered the chatroom but did not post a message. We recorded and analyzed how subjects responded to being studied. Results of a regression analysis indicate significantly more hostility in the three conditions where we said something than in the control condition. We were kicked out of $63.3 \%$ of the chatrooms we entered in the three message conditions compared with $29 \%$ of the chatrooms in the control condition. There were no significant differences between any of these three conditions. Notably, when given a chance to opt in, only 4 of 766 potential subjects chose to do so. Results also indicate significant effects for both size and the number of moderators. For every 13 additional people in a chatroom, the likelihood getting kicked out was cut in half. While legal and ethical concerns are distinct, we conclude by arguing that studying chatrooms constitutes human subjects research under U.S. law, but that a waiver of consent is appropriate in most cases as obtaining consent is impracticable.

Keywords chatrooms, ethics, informed consent, IRB, online research, waiver of consent

Learning what will in fact benefit may require exposing persons to risk. The problem posed by these imperatives is to decide when it is justifiable to seek certain benefits despite

Received 28 May 2003; accepted 4 September 2003.

We are grateful to those in the Electronic Learning Communities (ELC) research group and the Georgia Tech Institutional Review Board for valuable discussion in trying to conduct this type of research ethically. Thanks to Dave Robertson and Gilad Chen for advice on statistics and Sameer Patil for suggesting ICQ. Special thanks to Charles Ess for comments on an earlier draft. Thanks to Susan Herring, Brenda Danet, and two anonymous reviewers for their comments.

Address correspondence to James M. Hudson, College of Computing, Georgia Institute of Technology, Atlanta, GA 30332-0280, USA. E-mail: jhudson@cc.gatech.edu the risks involved, and when the benefits should be foregone because of the risks. (Department of Health, Education, and Welfare [DHEW], 1979, The Belmont Report, section B.2)

To determine whether a study involving human subjects should be carried out, we must weigh risks and benefits (DHEW, 1979, The Belmont Report). All research has some degree of risk. At minimum, the risks are comparable to those encountered in everyday life. At maximum, subjects, especially those in medical studies, may risk their lives. In any study, the degree of risk is weighed against the potential benefit of the study to both the individual and society. To complete this analysis, we must understand the nature of risks involved.

Starting in the early 1990s, the Internet grew from a tool used by a small population of specialists to a popular medium. Behavior of Internet users and accompanying changes in culture are of great interest to scholars from a wide variety of disciplines. Thoughtful research on this new medium can help us both understand its present and shape its future. However, such research must be conducted ethically, or we risk both harming individuals and also disturbing the very phenomena we seek to understand.

Research on the Internet raises a host of novel ethical challenges (e.g., Bassett \& O'Riordan, 2002; Boehlefeld, 1996; Bruckman, 2002; Ess, 2002; Eysenbach \& Till, 2001; Frankel \& Siang, 1999; Herring, 1996; King, 1996; Schrum, 1997; Walther, 2002; Waskul \& Douglass, 1996). Traditionally, research ethics relies on distinctions such as public versus private spaces, identified versus anonymous individuals, and published versus unpublished information. However, online, these categories become blurred (Bruckman, 2002; Eysenbach \& Till, 2001). Consequently, it can be difficult to translate our intuitions to the new domain of Internet research. Despite significant efforts from the American Psychological Association (Kraut et al., 2004), the American Association for the Advancement of Science (Frankel \& Siang, 1999), and the Association of Internet Research (Ess, 2002), many questions regarding the ethical conduct of online research remain. 
A host of particularly thorny ethical issues surround research on synchronous text-based computer-mediated communication or "chat." Is it ethical to enter a chatroom and record the conversation for research purposes? Under what circumstances? Is it necessary to obtain consent from participants? If so, what kind of consent? Is it sufficient to announce the researcher's presence and offer users a way to opt out of participation? Is it feasible to announce the researcher's presence but only record data if participants type a command to opt in? Is the process of obtaining consent more disruptive than the actual study? How should data collected from chatrooms be protected? Is it necessary to change pseudonyms of participants in written accounts? Is it acceptable to retain chatroom logs for long periods of time, or should they be coded for target behaviors and then destroyed to protect the privacy of participants? These are just a few of the difficult ethical questions this new medium raises.

These questions of course cannot be answered in the abstract, but must be addressed in the context of a particular set of research questions and methods. For example, a great deal more care was needed in Sheana Bull and Mary McFarlane's (2000) study of sexual behavior stemming from chatrooms catering to homosexuals than in Brenda Danet's (2001a) study of text-based art in IRC (Internet Relay Chat) chatrooms as a new form of folk art. Danet recorded activity in chatrooms in which individuals trade art derived from ASCII characters. In publishing on the subject, she included users' online pseudonyms unchanged, in order to give them credit for their creative work (Danet, 2001b). In this low-risk situation, not only were careful human subjects' protections not needed, but it could be argued that such protections would do her subjects a disservice by denying appropriate credit (Herring, 1996). In fact, in follow-up research, Danet's institutional review board (IRB) provided a complete waiver of consent (Danet, personal communication, 2003). In contrast, Bull and McFarlane recorded chatrooms that typically cater to homosexuals. They were interested in particular in evidence of online activity leading directly to risky face-toface behaviors. Before beginning their study, they first obtained a certificate of confidentiality-a legal document granting them the right to refuse to turn over their data even if faced with a court subpoena. After recording chatroom activity, they immediately coded the logs for instances of target behaviors - such as whether individuals made plans to meet face to face, and whether their HIV status was discussed in the course of making such plans. Once the frequency of such behaviors was coded and checked, the original logs were destroyed. These two studies at opposite ends of the risk spectrum necessitated quite different approaches to data management, retention, and publication.

In analyzing the ethical issues in any chatroom study, one key piece of information to understand is: How much do users object to being studied when they are aware of the study? In this article, we address this question empirically. Our intent is by no means to declare any particular type of work ethical or unethical, but simply to contribute concrete, empirically validated evidence to help researchers and IRBs make informed decisions. Of course, even with full knowledge of how users feel, difficult ethical decisions remain. In our discussion, we raise some of these issues.

Even if recording chatrooms is found to be extremely displeasing to users, that does not mean such research may not proceed. In fact, this study itself is an example of one that we could reasonably anticipate would annoy users greatly. However, after careful discussion with our IRB and specifying appropriate precautions to protect subjects, it was determined that the benefits outweighed the risks. Our goal in conducting this research is simply to help others make more informed decisions in planning future research.

\section{BACKGROUND}

In attempting to determine appropriate ethical conduct of Internet-based research, researchers tend to rely on a number of different metaphors for explaining the nature of the Internet. These metaphors, however, lead to different conclusions about whether or not individuals in Internet forums should be treated as human subjects. For some researchers, the Internet is like a public square, and for others, a private living room, a town hall meeting, or a newspaper letters column. Each of these metaphors leads to different ethical conclusions. In a public square, a researcher may observe behavior in a generalized way and write about aggregated results. In a private living room, permission of the participants is required for any research. A newspaper letters column does not require permission, but requires appropriate citations.

Relying on the metaphor that Internet chat is like a private space, many researchers take a perspective that individuals in chatrooms should be treated as human subjects. The human subjects approach to Internet research treats postings in online environments not as copyrighted statements, but as comments from individual subjects. Storm King (1996) argues that the same features that make Internet communication a worthy area of study also make it easier for a researcher to objectify the Internet-based subjects. As such, he proposes that Internet researchers must be cognizant of the fact that online contributors are, in fact, human subjects. When publishing research reports, King suggests that anonymity of both the subjects and the online forum itself are often necessary in order to protect all involved from harm. In this approach to Internet research, it is also necessary to obtain informed consent from the subjects of the research (Reid, 1996; Schrum, 1997). Exceptions occur, but the human subjects approach 
to ethical research assumes informed consent as a default position.

In keeping with a tradition of examining the reasonable expectations of potential research subjects (e.g., Elgesem, 2002; Walther, 2002), Elizabeth Bassett and Kathleen O'Riordan (2002) have suggested that examining the intent of those posting messages on the Internet might inform these ethical debates. In some spaces, online authors clearly use the Internet as a public forum in which to spread their ideas. Bassett and O'Riordan argue that human subjects protections (i.e., anonymity, informed consent, etc.) in these cases would do the authors a disservice and might even constitute unethical behavior. For example, when authors use the Internet to promote minority voices, such as alternative versions of newsworthy events, anonymization reinforces the dominant paradigm from which they are trying to escape. In other spaces, however, authors' comments clearly suggest that they consider the space to be relatively private. In such cases, human subjects protections might apply. As Bassett and O'Riordan point out, the challenge arises when different stakeholders suggest contradictory stances, such as when the operator of an online forum stresses the publicity and seeks to use the forum to promote a political cause while individual users treat it as a private space.

In the second author's previous work, Amy Bruckman (2002) suggests that the degree of potential harm might dictate how research is conducted and reported. In general, researchers work with dichotomies such as public versus private and published versus unpublished. Works on the Internet, however, turn these dichotomies into continua. Because these traditional distinctions blur online, she suggests that most online comments are semipublished and semipublic. This introduces a new dimension into the ethics of doing Internet research; there is a new continuum between individuals on the Internet deserving credit for their work and needing anonymity for protection. Bruckman concludes that a careful weighing of each of these dimensions in Internet research should suggest the amount of human subjects protections accorded to online subjects.

Coming from a view of online conversations as being like a newspaper letters column, some view online discussions as copyrighted material. In looking at U.S. law, for example, Edward Cavazos and Gavino Morin (1994, pp. 61-62) note:

\footnotetext{
If a chat participant is capturing the chat transcript, he or she is satisfying the fixing in a tangible medium required by the Copyright Act.... Users actively involved in on-line chat sessions probably should not consider their expressions protected unless those expressions are being transcribed, buffered, or captured to disk... The moment the writing, typing, or copying occurs, the copyright law covers the situation, giving the speakers immediate protection.
}

Since written materials on the Internet such as transcripts of chat sessions are considered copyrighted, they may be studied by academics within the bounds of fair use. Under this particular legal system, authors of such material do not have to consent to such use, nor do they have the right to withdraw consent. At the same time, however, researchers using such material may not anonymize their subjects in any way. When claiming "fair use," academics are legally required to cite their sources. If a researcher claims that consent is not necessary for fair use of material on the Internet, anonymizing the sources of such material is legally actionable. Cavazos and Morin do note, however, that these legal positions have not been clarified in court.

Susan Herring's (1996) critique of these arguments holds that they presuppose a specific research paradigm that excludes other legitimate types of research. In particular, none of these perspectives allows for linguistic research where the focus is on the form of the message, not the content. She concludes that research on the Internet is simply too broad to be covered by one set of ethical guidelines. Rather, each profession needs its own set of guidelines for dealing with Internet research. Only in this way is it possible for guidelines to account for the unique considerations of different approaches to research. Note that this perspective does not account for the fact that Internet researchers now often publish in multidisciplinary journals. As such, some minimal ethical guidelines are needed so that these different professions can appropriately talk to one another.

The study described in the next sections represents an attempt to systematically examine how potential subjects react to various methods of being studied. While one study cannot provide definitive answers to these difficult ethical questions, it can provide us with valuable insight. We conclude by reflecting on how these reactions alter the debate on the ethical conduct of research on the Internet.

\section{TERMINOLOGY}

Internet Relay Chat (IRC) is a protocol that allows individuals to communicate with one another through realtime text messages. While there are a number of ways to connect to IRC, a popular instant messenger client (ICQ $\left.{ }^{1}\right)$ provides a web-based interface that many less technologically aware individuals use. A number of chatrooms on any IRC server have a moderator, sometimes called an operator. The moderator has the ability to control many features of conversation in a given chatroom. For our purposes, the most important moderator ability is the ability to kick or boot someone out of the chatroom. When this happens, the individual who has been kicked out will immediately find himself or herself disconnected from that chatroom. The moderator can set an additional ban that will keep 
the booted individual from returning to the chatroom for a given time period.

For a number of reasons, bots are often used to aid moderators. Bots, where the name is derived from robots, connect to IRC like any other individual, but they are simply automated software programs that can be controlled by other (specified) individuals. From the perspective of individuals in the chatrooms, bots look like any other individual; there is nothing that distinguishes bots from people. When an authorized user sends a command to the bot, it will perform the specified action. Bots can also be programmed to perform various automated tasks.

\section{METHOD}

To understand how potential participants react to participating in online studies, we experimentally studied how individuals in online chatrooms reacted to a variety of conditions. We designed a study where we entered a number of online chatrooms, informed the participants that we were recording them to study language use, and recorded how individuals responded. Specifically, we examined participants in chatrooms on ICQ Chat. ${ }^{2}$ Since ICQ Chat uses IRC servers, we were able to conduct this study without worrying about proprietary software (such as MSN Chat). Also, ICQ Chat's web-based interface offered a population that is generally less technologically aware than standard IRC populations. Because of this web-based interface, we have reason to believe that individuals using ICQ Chat are more representative of the general population of Internet users than those on most other IRC servers.

First, we downloaded a list of the available chatrooms each evening at 9:50 p.m. ${ }^{3}$ On any given day, the mean size of available chatrooms on most IRC networks tends to be positively skewed: There are a large number of small chatrooms, but fewer large ones. Figure 1 illustrates the distribution of chatrooms on one typical day in our study. Note that a couple of the larger chatrooms-those with more than 100 participants - are not pictured for the sake of visual clarity. In order to ensure that we adequately covered the range of potential chatroom sizes, we arbitrarily divided the available chatrooms into four buckets: very small (2-4 participants), small (5-10 participants), medium (10-29 participants), and large (30 or more participants). This means we sampled a much larger percentage of the available large chatrooms than of the available smaller chatrooms.

Using these buckets, we randomly chose 16 chatrooms from each. Each set of 16 chatrooms was further (randomly) subdivided into groups of 4 . Each group of four was assigned to one of our recording conditions. In each condition, we varied the message we said to the chatroom. In the No Message condition, we simply entered using the nickname "Chat_Study" and said nothing. In the Recording

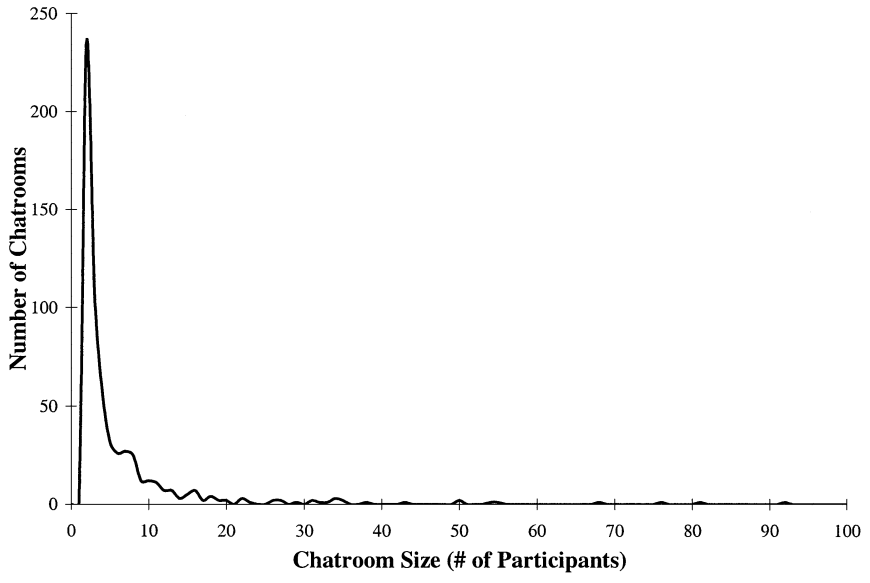

FIG. 1. Distribution of chatrooms on a typical day at 10:00 p.m. Note: A few chatrooms with more than 100 participants have been removed from this figure for the sake of visual clarity.

Message condition, we entered as "Chat_Study" but announced that we were recording the chatroom for a study. The Opt In Message and Opt Out Message conditions were similar, but allowed individuals to choose to opt in or opt out of the study by typing a response. The exact messages used are listed in Table 1.

Once chatrooms were randomly assigned to conditions, we entered the chatrooms (in a random order) and conducted the study. Upon joining a room, we waited 1 minute before posting our message. Then, we waited another 5 minutes before leaving the chatroom. If we had not been kicked out of the chatroom by this time, we posted the following message before exiting:

This research is actually not about language use. Rather, it is designed to study how individuals in chatrooms react to researchers studying them. Further information is available at http://www.cc.gatech.edu/elc/chatstudy. Thanks!

By entering chatrooms two at a time and staggering our conditions, we were able to test 64 chatrooms within a 1-hour period (10:00 p.m.-11:00 p.m.).

For each chatroom, we noted the number of participants at the time we entered, whether or not a moderator was present, whether or not conversation occurred, and whether or not we were kicked out of the room. If a chatroom did not have a moderator or we did not observe conversation, it was removed from the study prior to data analysis. Running this study each evening from 1 March until 14 March 2003, ${ }^{4}$ we sampled 525 chatrooms. Of these, we retained the 137 chatrooms with moderators and active conversation for our data analysis. Figure 2 presents a rough breakdown of the topics under discussion, based on the name of the chatroom. We later present both quantitative and qualitative analyses of how individuals in these chatrooms responded to us. 
TABLE 1

Announcement messages

\begin{tabular}{|c|c|}
\hline Condition & Message broadcast \\
\hline No message & None \\
\hline Recording message & $\begin{array}{l}\text { We are researchers recording this chatroom for a study on language use in online environments. For } \\
\text { questions or comments, email study@mail.chatstudy.cc.gatech.edu. Thank you! }\end{array}$ \\
\hline Opt out message & $\begin{array}{l}\text { We are researchers recording this chatroom for a study on language use in online environments. If you } \\
\text { don’t want to be recorded, please whisper "Chatstudy opt out” to us. For questions or comments, email } \\
\text { study@mail.chatstudy.cc.gatech.edu. Thank you! }\end{array}$ \\
\hline
\end{tabular}

Note. The "study on language use" was chosen as a specific innocuous study.

\section{Ethical Issues}

Before we delve into the results, there were a number of ethical issues that arose in the design and conduct of this study. In essence, this is a deceptive study conducted on 2260 subjects $^{5}$ without their consent. In conducting this research, we decided to work under the most restrictive of the proposed ethical stances-the human subjects model. As such, we sought permission from Georgia Tech's Institutional Review Board (IRB) for conducting this research. Our IRB had three primary concerns in reviewing this research: the use of deception, the lack of consent, and the potential for harm.

Responding to concerns over the potential for harm is quite difficult in a study designed to evaluate the potential for harm in studies like it. Since most reported cases of significant harm as a result of this type of research have involved conversations about sensitive topics (Bassett \& O'Riordan, 2002; Reid, 1996), we agreed to review all po-

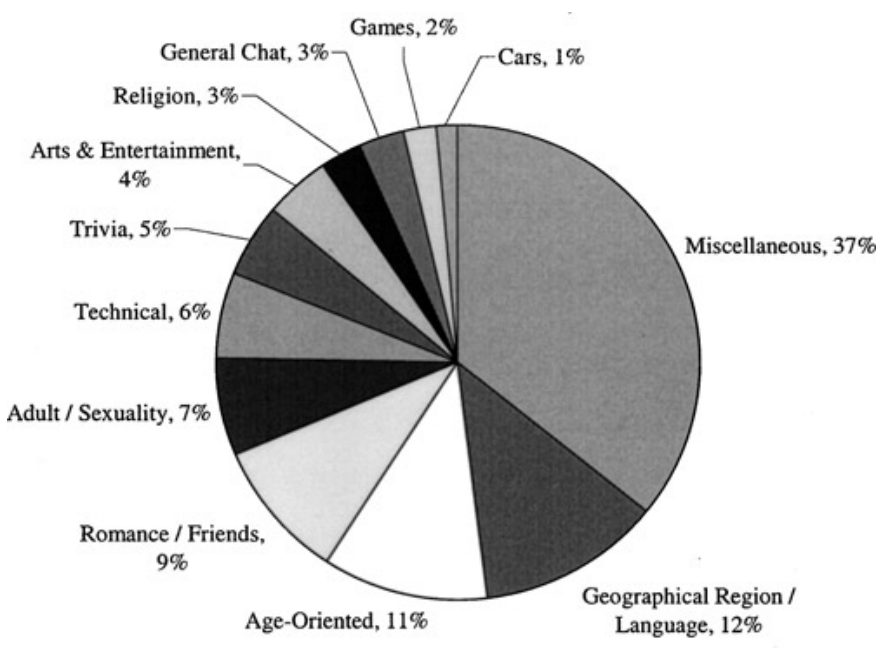

FIG. 2. Distribution of chatrooms in our study. tential chatrooms before entering them to ensure that sensitive discussions seemed unlikely. While we never formally defined what we meant by "sensitive topics," we used emotional support groups such as "breast cancer survivors" as the prototypical discussions to avoid. In conducting the study, we encountered no such chatrooms. While we do not provide specific chatrooms names here, Figure 2 shows the general types of chatrooms encountered. To further minimize harm, we agreed to limit the scope of our study to only comments directly pertaining to us. Specifically, after reading through the transcripts once, we removed all comments that were not directed to or about us. All data analysis was performed on these cleaned transcripts.

Generally in deception-based research, subjects consent to participate in a research study, but are deceived about the exact nature of the research (e.g., Milgram, 1974; Latané \& Darley, 1970). When this is justified, subjects should be debriefed to the extent possible about the true nature of the research. To do so, we pointed subjects to a web page with information about our study before we left the chatroom. We decided, with the help of our IRB, that we would not debrief chatrooms where we had been kicked out. Decisions about debriefing in this type of study involve balancing subjects' right to be debriefed with their right to be left alone. Since kicking us out of the chatroom would indicate a strong desire to be left alone, we gave this right greater weight. We felt that the additional disruption would cause more harm than the benefit that debriefing provided.

Our study had an additional challenge in that we were not getting consent from subjects to participate in the study at all. Based on the U.S. federal regulations governing research, informed consent may be waived only when four conditions are met:

1. The research involves no more than minimal risk to the subjects.

2. The waiver or alteration will not adversely affect the rights and welfare of the subjects. 
3. The research could not practicably be carried out without the waiver or alteration.

4. Whenever appropriate, the subjects will be provided with additional pertinent information after participation (45 CFR 46.116.d).

After much discussion, our IRB felt that we met all four of these conditions and qualified for a waiver of informed consent. More discussion on waivers of consent is provided in our data analysis.

\section{A Note on Generalizability}

Like all research studies, we must deal with a number of questions of generalizability. Do our findings from ICQ Chat generalize to other chat environments such as EFnet, DALnet, or MSN Chat? How might our findings from chat environments generalize to other online environments? Do our methods generalize to communities where researchers are already a member of the community being studied?

In choosing a specific site for this research, we wanted to meet several criteria. We wanted a chat environment with a large enough base of users to obtain a reasonable sample size. At the same time, however, we wanted the participants' backgrounds to be as similar to general Internet users as possible. Since IRC users tend to be more technologically savvy than mainstream Internet users, this limited us to ICQ Chat, MSN Chat, and Yahoo! Chat. Since it was important for us to automate our data collection, we had to avoid chat systems with proprietary chat software. This left us with ICQ Chat. The similarities between all of these text-based chat environments, however, lead us to believe that findings from ICQ Chat will likely largely generalize to other such spaces.

We believe that our findings will likely generalize to other types of online environments to the extent that they are similar to chat environments. The Internet is not monolithic; many different types of online spaces exist. Some focus more on chat-style discussions but use avatars (e.g., the Palace, EverQuest, Ultima Online) or audio instead of text (e.g., X-box Live). Some environments remain in text, but do not feature synchronous interaction (e.g., newsgroups, message boards). Others contain more identifying information such as pictures (e.g., personal webpages), personal stories (e.g., blogs), or video (e.g., CUSeeMe).
While we expect our findings to generalize to some of these environments, we cannot predict to what extent without further studies.

There are also questions about how well this research method will extend to other means of data collection. In anthropological work, there are two basic types of research strategies: emic and etic (Headland et al., 1990). In emic research, it is the interpretation of those being studied that is most valued (Rosaldo, 1989). Often, emic research occurs in online environments when a researcher realizes that there is something scientifically interesting about a community he or she is part of. In this case, the researcher approaches studying the community as an insider. In etic research, however, the interpretation of the scientific community is more valued than that of the community members (Rosaldo, 1989). Researchers from this perspective approach a community as an outsider. They announce their affiliation as researchers from the outset of their research. In the study described here, we are engaging in the most prototypical type of etic research. How these findings generalize to emic methods is debatable. Studies conducted by true community insiders still raise a host of ethical issues, but those issues are likely to differ substantially from those described in this paper. Once again, more research is needed to sort out all of these complicated ethical issues.

\section{RESULTS}

The results of this study are divided into two parts. In the first section, we perform a quantitative analysis of the factors that influence whether or not we were kicked out of a chatroom. The second section presents a more qualitative analysis of comments to or about us. Table 2 presents descriptive statistics about each of our conditions. A oneway analysis of variance (ANOVA) analysis on the average size of a chatroom in each condition revealed that there were no significant differences between the conditions $\left(F_{(3,133)}=0.513, p=.674\right)$.

\section{Quantitative Analysis}

To analyze which factors contributed to whether or not we were kicked out of the chatrooms, we conducted a hierarchical logistic regression analysis. Our dependent

TABLE 2

Descriptive statistics of conditions

\begin{tabular}{lcccccc}
\hline Broadcast message & $\mathrm{n}$ & Mean size & Minimum size & Maximum size & Standard deviation & Kicked frequency \\
\hline None & 28 & 17.36 & 2 & 139 & 27.542 & $29 \%$ \\
Recording & 34 & 16.62 & 2 & 99 & 21.061 & $56 \%$ \\
Opt out & 36 & 12.31 & 2 & 46 & 10.485 & $72 \%$ \\
Opt in & 39 & 19.64 & 2 & 201 & 36.604 & $62 \%$ \\
\hline
\end{tabular}


TABLE 3

Contrast coding for broadcast message

\begin{tabular}{lccc}
\hline $\begin{array}{c}\text { Broadcast } \\
\text { message }\end{array}$ & $\begin{array}{c}\text { Contrast 1: } \\
\text { vo message }\end{array}$ & $\begin{array}{c}\text { Contrast 2: } \\
\text { vsnounce and opt out } \\
\text { vs. opt in }\end{array}$ & $\begin{array}{c}\text { Contrast 3: } \\
\text { Announce } \\
\text { vs. opt out }\end{array}$ \\
\hline None & 1 & 0 & 0 \\
Recording & $-1 / 3$ & $1 / 2$ & 1 \\
Opt out & $-1 / 3$ & $1 / 2$ & -1 \\
Opt in & $-1 / 3$ & -1 & 0 \\
\hline
\end{tabular}

variable was whether or not we were kicked out of the chatroom. (A "1" indicated that we were kicked out.) The first step of the analysis looked at possible order effects based on the day that we collected data. Since this study was spread over 2 weeks, this step attempted to control for cross-pollution that might result from word of our presence getting around. In the second step of the analysis, we introduced two additional variables: the size of the chatroom and the number of moderators in the chatroom. With the number of moderators, we were interested in measuring the number of individuals who could potentially kick us out of the chatroom. However, since chatrooms often have bots with operator status and individuals who can use these bots to obtain operator status, this is an imperfect measure. In the final step of the regression analysis, we introduced our experimental condition in the form of three contrast coded variables. These contrasts are listed in Table 3. Recall that contrast coding of four experimental conditions requires three orthogonal contrasts. Therefore, while our third contrast (between the recording message condition and the opt out condition) is not a hypothesis that we were explicitly interested in, it is required for this type of statistical analysis. Table 4 presents our correlation matrix. Table 5 presents the results of our analysis.

Results from this analysis indicate that both size $\left(\operatorname{Wald}_{(1)}=5.407, p=.020\right)$ and the number of mod- erators $\left(\operatorname{Wald}_{(1)}=7.491, p=.006\right)$ significantly predicted when we were kicked out of chatrooms. Briefly, the likelihood of being kicked out of a chatroom decreased as the number of people present increased. We were twice as likely to be kicked out of a room with 5 people than a room with 18 people, holding other factors constant; for every 13 additional people in a chatroom, the chances of being kicked out were cut in half. Conversely, increased numbers of moderators lead to increased chances of being kicked out.

In addition, our experimental conditions are significant predictors $\left(\chi_{(3,137)}^{2}=15.554, p=.001\right)$ over and above all other variables. The No Message condition is significantly different from the other three conditions (Contrast 1: Wald $\left._{(1)}=12.286, p<.001\right)$, but there were no other differences found between conditions. In other words, it did not matter what we said. Any indication of recording the chatroom significantly increased our likelihood of being kicked out. In fact, holding other variables constant, we were nearly four times more likely to be kicked out when we said something.

\section{Qualitative Analysis}

When We Were Kicked Out. In IRC, when an operator chooses to kick someone out of the chatroom, he or she has the option of providing additional text to explain why that decision was made. Since everyone in the chatroom can see this message, any text is written as much for the members of the chatroom as it is for the individual kicked out. When we examine the messages explaining why we were kicked out in this study, we see a number of general themes: prohibitions against spamming, opposition to being studied, general requests to leave, and insults.

Of the 77 times that we were kicked out, 13 messages (17\%) explicitly referred to our study as spamming:

- "You're being banned for 43200 minutes, for spamming."

TABLE 4

Correlation matrix

\begin{tabular}{lrrrrrr}
\hline & Order & Size & $\begin{array}{c}\text { Number of } \\
\text { moderators }\end{array}$ & $\begin{array}{r}\text { Condition } \\
\text { Contrast 1 }\end{array}$ & $\begin{array}{r}\text { Condition } \\
\text { Contrast 2 }\end{array}$ & $\begin{array}{r}\text { Condition } \\
\text { Contrast 3 }\end{array}$ \\
\hline Order & 1.000 & & & & & \\
Size & 0.465 & 1.000 & & & & \\
Number of moderators & 0.012 & -0.287 & 1.000 & & & \\
Condition Contrast 1 & -0.009 & 0.124 & -0.206 & 1.000 & & \\
Condition Contrast 2 & 0.047 & 0.008 & -0.030 & -0.019 & 1.000 & \\
Condition Contrast 3 & -0.123 & -0.007 & -0.110 & 0.038 & -0.025 & 1.000 \\
\hline
\end{tabular}

Note. All correlations are relatively small. Therefore, multicollinearity does not present a problem in this analysis (Pedhazur, 1997). 
TABLE 5

Regression results

Step 1: Controlling for Order Effects. Did the day we ran the study on affect whether or not we were kicked out of the rooms? Effects were found not significant

\begin{tabular}{lcccccc}
\hline Variable & B & Wald & $\operatorname{Exp}(\mathrm{B})$ & Percentage correct & $\Delta \chi_{(1)}^{2}$ & $\chi_{(1)}^{2}$ \\
\hline Order & 0.072 & 2.136 & 1.075 & $62.8 \%$ & 2.180 & 2.180 \\
\hline
\end{tabular}

Step 2: Controlling for Size and Moderators. Did the size of the chatroom affect whether or not we were kicked out of the rooms? Did the number of moderators affect whether or not we were kicked out? Effects for both cases were found significant

\begin{tabular}{lclllll}
\hline Variable & B & Wald & $\operatorname{Exp}(\mathrm{B})$ & Percentage correct & $\Delta \chi_{(2)}^{2}$ & $\chi_{(3)}^{2}$ \\
\hline Order & -0.002 & 0.001 & 0.998 & & & \\
Size & $-0.048^{*}$ & $5.348^{*}$ & $0.0953^{*}$ & & & \\
Number of moderators & $0.090^{*}$ & $5.502^{*}$ & $1.094^{*}$ & $67.2 \%$ & $16.202^{* *}$ & $18.381^{* *}$ \\
\hline
\end{tabular}

Step 3: Evaluating Message Conditions. Were there any differences between any of our conditions? Significant effects were found for the difference between saying something and saying nothing, but no other differences existed

\begin{tabular}{lcclccc}
\hline Variable & $\mathrm{B}$ & Wald & $\operatorname{Exp}(\mathrm{B})$ & Percentage correct & $\Delta \chi_{(3)}^{2}$ & $\chi_{(6)}^{2}$ \\
\hline Order & 0.008 & 0.016 & 1.008 & & & \\
Size & $-0.055^{*}$ & $5.407^{*}$ & $0.947^{*}$ & & & \\
Number of moderators & $0.113^{*}$ & $7.491^{*}$ & $1.119^{*}$ & & & \\
Condition Contrast 1 & $-1.335^{* *}$ & $12.286^{* *}$ & $0.263^{* *}$ & & & \\
Condition Contrast 2 & 0.013 & 0.002 & 1.013 & & & \\
Condition Contrast 3 & 0.106 & 1.684 & 0.700 & $69.3 \%$ & $354^{* *}$ & $33.936^{* *}$ \\
\hline
\end{tabular}

Note. Significance: ${ }^{*} p<.05 ;{ }^{* *} p<.001$.

- "Go Advertise Somewhere Else"

- "do not advertise here"

- "no spamming"

- "spam"

- "Don't fucking advertise"

- "spam"

- "lame ass spamm... get a life"

- "No spamming."

- "don't spam"

- "no spammers thnx"

- "Advertisment Kick $\rangle$ No advertising in \#〈deleted $\rangle "$

- "no spam!"

Note that each chatroom only saw our message once. Any suggestion of "spam" comes from either the content or length of our message. Herring's (1999) research on one chat server, for example, suggests that most chatroom messages are significantly shorter than the one we posted.

Ten other chatrooms (13\%) explicitly voiced opposition to being studied:

- "no"

- "how bout no"
- "no, thank you!"

- "we dont do studies"

- "No studying"

- "No, dammit!!"

- "This is a private room, no studies needed"

- "No thanks, we gave at the office."

- "study somewhere else"

- "That behaviuor is not allowed in here - You can leave now !!"

For these chatrooms, it is clear that the concept of being studied was antagonistic. The comments explicitly express a desire not to be studied.

Several kick messages expressed a desire for us to leave the room:

- "go away!!!!!!!!!”

- "go away,"

- "bye bye dont bother us"

- "You are frightening our customers, we must ask you to leave"

- "Bye!"

- "I SAID SHOO"

- "soo long duud" 
Others simply offered a variety of insulting remarks:
- "Would you like fries with that?"
- "oh please ...u can kiss my ass :)"
- "Excuse me...I'd like to ASS you a few ques- tions!!!"
- “... study this..."
- "... and there was much rejoicing..."
- "Yo momma so ugly she turned Medusa to stone!"
- "I'm only doing this because I care"
- "Quit flappin your lips, know your role beeyatch"
- "Talk to the foot!"
- "Oh! Oh! Kick me again! - tempban 1 hr"
- "A Fast One, Like It?!?!"
- "Boot to the head!"

The remaining comments were simply descriptive (e.g., "Chat_Study is banned (u are not welcome or wanted byessss)") or irrelevant (e.g., "I'm too lazy to give you a real kick message.”).

When We Were Not Kicked Out. Of the 60 times that we were not kicked out of the chatrooms, 20 (33\%) of them were in the No Message condition. In most of these cases, the participants gave no indication that they were aware of our study. Sometimes individuals greeted us, but usually we were ignored. In the 40 cases where subjects were aware that we were studying them, however, we only noted one pattern: Trivia chatrooms tend not to kick us out. In these chatrooms, a bot asks trivia questions for the audience of participants to answer. The bot is able to determine who gets the questions right and award the appropriate points. Eight of the 11 trivia chatrooms did not kick us out. Of these, however, three were in the No Message condition. While this is far from conclusive, it suggests that the attitudes of participants in trivia chats might be somewhat different from those in other chatrooms.

Opting In and Opting Out. Based on the results from the Opt In and Opt Out conditions, there is little reason to believe that these are viable ways of conducting research. In the Opt Out condition, we were kicked out of the chatrooms $72 \%$ of the time. With Opt In, it was $62 \%$ of the time. (There is no significant difference between these two conditions.) Of the 443 individuals who could have responded in the Opt Out condition, only two individuals opted out. A few others, however, did express what might be called a desire to opt out (e.g., "hey chat fuk off," "yeah up urs chatstudy!!"). Of the 766 individuals in the Opt In condition, only four chose to do so. Even in this condition, some individuals expressed strong disagreement with the possibility of being recorded (e.g., "please leave Chat_Study u do not have permission ... now all we need is for Chat_Study to fuck Off."). For the most part, however, the negative comments we received in these two conditions were less frequent and less vehement than those we received in the Recording condition (e.g., “〈deleted〉 kicks Chat_Study's ass so hard.... Chat_Study will be shitting out of it forhead for a week!", "Hey Chat_Study dont you ever talk to me like that again you fucking flaccid, nasty, skank ugly, idiotic no brained, small dicked, stinking nasty pimp daddy wannabe, go wave that limp nasty scab encrusted dick somewhere else bitch!').

\section{DISCUSSION}

Based on this study, we can safely conclude that individuals in online environments such as chatrooms generally do not approve of being studied without their consent. The vehement reaction of many in our study indicates that they object to being studied. Further, when given the option to opt in or opt out of research, potential subjects still object. The fundamental limitation of these data, however, is that we cannot distinguish between those who are opposed to being studied and those who are opposed to the consent process. In some of the kick messages, there's an indication that subjects were opposed to being studied (e.g., "This is a private room, no studies needed"). On the other hand, the high level of hostility in the Opt In condition suggests that the consent process caused some of the response we observed. We should note that an alternative explanation holds that the potential subjects in the Opt In condition (correctly) did not trust us to record them only with explicit consent. Further research is needed to be able to distinguish between these confounds.

These data raise a number of tricky questions. What exactly do subjects' reactions indicate about their thoughts or feelings? Even if we could say that subjects perceived harm in this study, what should we do about it? We cannot know based on solely this study. Without further research, the answers are disputable. We can, however, say that these data suggest it is impracticable to obtain consent for studying behavior in chatrooms. In conditions where we informed subjects of our study, they kicked us out of the chatrooms nearly two-thirds of the time. Regardless of whether we were kicked out of the chatroom, many individuals reacted with hostile statements. When given the option to opt in to our study, only 4 individuals out of 766 potential subjects chose to do so. Opting in to the study-the closest analog to traditional informed consent-was clearly not viable in this study. While we cannot draw conclusions from one study, these results suggest that obtaining consent for studying online chatrooms is impracticable. Of course, further research that manipulates many of the details of this study is necessary to understand the nuances involved in obtaining consent in this type of environment.

This leads us to the question: If subjects are not aware that a researcher is recording the conversation in a chatroom, is there still harm? A teleological perspective such 
as utilitarianism holds that no harm has been done (Mill, 1998). The subject unaware of research cannot feel disrupted or harmed. Therefore, the benefits of the situation outweigh the potential for harm. It is important to note that this line of ethical reasoning hinges on the (arguably tenuous) assumption that subjects will never become aware of the research. A more deontological perspective holds that there are certain rights that are fundamental (Kant, 1981). As The Belmont Report (DHEW, 1979) states:

Respect for persons requires that subjects, to the degree that they are capable, be given the opportunity to choose what shall or shall not happen to them.... An agreement to participate in research constitutes a valid consent only if voluntarily given. (Part C.1)

A violation of these rights, whether or not the subject is aware of the violation, constitutes harm. Therefore, violating a subject's right to consent to participate in a study is harm even if the subject is unaware that the right has been violated.

Tied in with this question, we must ask about the ethics of harming potential subjects through the consent process. Our data indicates that chatroom participants kicked us out roughly two-thirds of the time when we attempted to obtain informed consent. Which is the greater harm-annoying two-thirds of potential subjects or not obtaining consent? Once again, this is a difficult question where reasonable people can disagree.

While we cannot seek to solve these ethical problems in this article, the results of this study lead us to believe that research in preexisting chatrooms can be conducted most productively when subjects are unaware of the study. Individual researchers must decide for themselves whether or not it is ethically right to do so. Given this conclusion, however, there are three ways under the U.S. ${ }^{6}$ regulations governing academic research that we can go about doing research without the consent of potential subjects: (a) Determine that the research is not human subjects research, (b) determine that the research is exempt from IRB oversight, or (c) convince an IRB to issue a formal waiver of consent. As we will show, the first two of these approaches are problematic. Assuming that a researcher has decided it is ethically appropriate to conduct a given study without obtaining subjects' consent, we conclude that obtaining a waiver of consent from an IRB is the most appropriate way to conduct chatroom research under U.S. regulatory law.

In exploring the issue of consent, we should also consider appropriate means of data protection. Should log files be anonymized or not? Should they be kept at all? For how long? Are ethnographic-style field notes more appropriate? Certainly, there is no one answer, but these issues affect the degree of potential harm. Different levels of data protection are required for different studies depending on both the sensitivity of the discussion and the research ques- tions asked. As we discuss the human subjects issues next, we highlight some appropriate types of data protection.

It is our belief that, with few exceptions, chatroom research classifies as human subjects research. The federal guidelines define a human subject as:

Human subject means a living individual about whom an investigator (whether professional or student) conducting research obtains:

(1) Data through intervention or interaction with the individual, or

(2) Identifiable private information. (45 CFR 46.102.f)

Research on chatrooms becomes human subjects research as soon as the researcher interacts with the participants. Clearly, this includes any form of participant observation. Even without interaction, though, most observation of chatrooms is human subjects research because the logging of conversations generally involves collecting identifiable private information. The researcher doing this type of work collects pseudonyms and, potentially, IP addresses of each participant. The American Association for the Advancement of Science (AAAS) report on online research points out that pseudonyms both function as real names and often can be linked to real names (Frankel \& Siang, 1999). While some researchers debate the ease with which pseudonyms and real names can be linked, it is hard to know a priori the difficulty of such linking. Likewise, IP addresses can often be traced back to individual computers (Nosek et al., 2002). In fact, new federal regulations on the protection of health information (HIPAA) specifically define IP addresses as identifying information (45 CFR 160.514.b.2.i.O). It is reasonable to assume that this same definition would be applied to other types of research. Therefore, it is our belief that this information does constitute "identifiable private information." As such, observational research on chatrooms constitutes human subjects research.

It is important to note that the definition of human subjects research does not consider the nature of the research questions being asked. In fact, the specific research questions are irrelevant for determining if a project constitutes human subjects research. Note that the formal definition of "research"- - "a systematic investigation ... designed to develop or contribute to generalizable knowledge" (45 CFR 46.102.d) — does not deal with the nature of the specific research questions either. This issue, however, is considered in determining the benefits and risks of a study when considering the possibility of exemption or waivers of consent.

For human subjects research, it is often possible for an IRB to declare research exempt from IRB oversight. Of the possible categories for exemption, one is relevant to this type of research: 
Research involving the use of educational tests (cognitive, diagnostic, aptitude achievement), survey procedures, interview procedures or observation of public behavior, unless:

(i) Information obtained is recorded in such a manner that human subjects can be identified, directly or through identifiers linked to the subjects; and

(ii) any disclosure of the human subjects' responses outside the research could reasonably place the subjects at risk of criminal or civil liability or be damaging to the subjects' financial standing, employability, or reputation. (45 CFR 46.101.b.2, emphasis added)

Note that decisions regarding exemption are the sole purview of the IRB and should not be made by researchers alone.

In general, this exemption will not apply to chatroom research since the potential for harm is unclear. When the topics of discussion online are sensitive, the potential for harm is clear and strong data protection measures appropriate. In others, though, harm tends to arise in unexpected ways. For example, records of an online flirtation may be subpoenaed in a divorce case. While the potential for harm can never be fully determined in advance, the question should be discussed with the IRB and appropriate data protection should be considered. Researchers interested in general behavioral questions might keep transcripts for only a short time period while field notes are made. Researchers interested primarily in the form of messages might want to remove identifiers from the transcript. Specific details are tricky and should be discussed with the IRB.

The American Heritage Dictionary of the English Language (2000) defines privacy as "the state of being free from unsanctioned intrusion." While chatrooms are considered by many to be public spaces, this study provides evidence that many participants consider them private spaces, which influences the potential for harm (King, 1996). Clearly, many subjects felt that our presence as researchers in the chatroom constituted "unsanctioned intrusion." As Eysenbach and Till (2001) hypothesized, the significance of size in our regression analysis indicates that individuals in smaller chatrooms are more likely to perceive the space as private than those in larger chatrooms. While smaller chatrooms are publicly accessible, research methods should account for these greater feelings of privacy. ${ }^{7}$ Chatrooms are publicly accessible spaces, but researchers must be sensitive to subjects' perceptions of privacy when choosing appropriate research methods.

The final potential harm that researchers and IRBs should discuss is the possibility of minors as research subjects. In chatrooms, it is usually difficult or impossible to ascertain the age of potential research subjects. Combined with the growing number of children and teenagers using the Internet, this implies that often minors will be involved in chatroom research. Although the name of a chatroom might give some indication about the expected age of the participants, there is usually no clear way to exclude minors from chatroom research. Since research involving minors generally requires more stringent subject protection, researchers and IRBs should carefully discuss the implications of minors in an online study.

Given this list of challenges in conducting Internet research, most IRBs will likely feel uncomfortable giving exemption from oversight. Researchers will often need to obtain a waiver of consent to conduct research in chatrooms without the participants' knowledge. As we discussed earlier, waiving informed consent requires that four conditions be met by the research:

1. The research involves no more than minimal risk to the subjects.

2. The waiver or alteration will not adversely affect the rights and welfare of the subjects.

3. The research could not practicably be carried out without the waiver or alteration.

4. Whenever appropriate, the subjects will be provided with additional pertinent information after participation. (45 CFR 46.116.d)

A researcher requesting a waiver of informed consent will need to explicitly deal with each of these four conditions. The results of this study provide specific evidence that most studies in chatrooms could not be practicably carried out without a waiver of consent. Note that IRBs granting waivers of consent will require regular review of the protocol. Typically, IRBs will require annual review- the maximum time allowed by U.S. regulations. It is possible, however, for IRBs to require shorter review cycles. In this particular study, for example, the Georgia Tech IRB required that we submit information to review every three months.

When obtaining a waiver of consent for sensitive discussions, the most stringent data protection will be necessary. If researchers need to maintain transcripts of online conversations, such as in linguistics studies, it will be necessary to remove all identifying information as soon as possible. When exact transcripts are not necessary, however, researchers might maintain the transcripts for a short period of time while documenting ethnographic-style field notes (e.g., Bull \& McFarlane, 2000). After the notes are written, the transcripts can be destroyed. An alternative approach is to create a chatroom specifically for research purposes (e.g., Hudson \& Bruckman, 2002). Creating a new chatroom allows researchers to set expectations so that subjects are aware of being studied. Since there is a range of ethically defensible responses, depending on the details of the study design, potential participants, etc., discussions between researchers and the IRB can help clarify some of the potential issues and their resolutions. 


\section{CONCLUSION}

The findings of this study suggest that individuals in chatrooms react with hostility when they are aware of being studied. In the three conditions where subjects were explicitly aware of our study, we were kicked out of $63 \%$ of the chatrooms. When subjects only had our name ("Chat Study") as evidence of our research, however, we were only kicked out of $29 \%$ of the chatrooms. There were no significant differences in being kicked out depending on what we specifically said. Notably, when given the chance to opt in, only 4 out of 766 potential individuals chose to do so.

Results also suggest that the level of perceived privacy in chatrooms varies based on the size of the chatroom. In smaller chatrooms, we experienced significantly greater hostility than in larger chatrooms. In fact, for every 13 additional individuals in the chatroom, the chances of being kicked out were cut in half. Further research is necessary to understand the source of this perception of privacy - for example, issues of control, insider-outsider dynamics, or other alternative explanations.

These findings lead to the conclusion that obtaining consent in investigating chatroom environments is impracticable. This conclusion, however, raises difficult questions: Is it ethically right to study chatroom participants without their consent? Is it right to annoy/disturb significant numbers of participants in order to obtain consent from a few? The answers are not clear. Different ethical frameworks lead to different conclusions. For example, a utilitarian framework weighs the benefits and harms of a given course of action. If the subject will not experience any concrete harm, the benefits ethically justify carrying out the research without a subject's consent. A deontological framework, however, holds that individuals have certain rights that cannot be violated without causing harm. Even though the subject is unaware of the violation, conducting research without his or her consent causes harm. The challenge in doing ethical research often involves making difficult choices about competing ethical claims (DHEW, 1979, The Belmont Report). Is it ethically acceptable to forgo an individual's right to consent to research in order to further scientific understanding that might provide many benefits? Competing ethical claims make this a difficult issue. In offering guidelines for resolving these difficult ethical dilemmas, The Belmont Report (DHEW, 1979) takes an explicitly utilitarian perspective, urging researchers to weigh the potential risks and benefits of any given research project on a case-by-case basis.

While we cannot answer these questions here, one thing seems clear: the details matter. When researchers discuss these types of studies with their IRBs, they must keep in mind several important questions:
- Consent: Is there reason to believe that obtaining consent will be difficult? Will the process of requesting consent itself cause harm? Is it possible to obtain consent in some other way (e.g., create a special chatroom explicitly for the study)?

- Harm: What are the potential harms in conducting the study? What if someone links a subject with this study? Will this study deal with sensitive topics (e.g., health support, illegal activities, etc.)? Is there reason to believe that children might be subjects?

- Data protection and retention: How long are data records maintained? Who will have access to these records?

- Anonymization: What types of identifiers exist in the data record? Real names? Pseudonyms? IP addresses? Will the researchers remove these identifiers before data analysis? If so, how long will it take before identifiers are removed?

- Credit: Are the subjects likely to deserve or desire credit for their work? Will subjects feel their copyright has been violated if they are anonymized in research publications?

Answers to these questions influence the potential for harm in any given study. As such, researchers and IRBs must carefully consider these issues.

In this article, we have provided evidence that it is probably infeasible to study preexisting chatrooms as an outsider while also obtaining informed consent from the participants. This offers evidence that waivers of consent may be appropriate for this type of research, provided (1) that the other criteria for waivers of consent are met and (2) that the researcher decides that research without consent is ethically defensible. When an IRB is considering granting such a waiver, however, it is important to carefully consider issues of data protection and retention. Since there are no easy answers in obtaining and analyzing data, considerations should occur on a case-by-case basis through dialog between researchers and IRBs.

\section{NOTES}

1. http://www.icq.com.

2. http://www.icq.com/ircqnet.

3. Note that all times are Eastern Standard Time.

4. Due to technical problems with the software and the network respectively, no data were collected for Tuesday 4 March or Friday 14 March.

5. This represents the number of unique usernames involved in our study. There is not necessarily a one-to-one mapping between individuals and user names.

6. Since our personal research experience has occurred in the United States, we focus on U.S. laws. The first author (Hudson) is a member of Georgia Tech's Institutional Review Board (IRB). The second author 
(Bruckman) has served on a number of panels on the ethics of Internet research, including the American Psychological Association (APA), the American Association for the Advancement of Science (AAAS), and the Association for Internet Research (AoIR). In focusing on U.S. law, we acknowledge that legal considerations are often not the same as ethical considerations. We also note that U.S. law differs substantially from other legal systems and that international requirements may vary.

7. This issue comes up in nonelectronic research contexts. It's debatable how subjects would respond if we did this same study at the local coffee shop. The issues are complex and must be understood on a case-by-case basis.

\section{REFERENCES}

American Heritage Dictionary of the English Language. 2000. 4th ed. Boston: Houghton Mifflin.

Bassett, Elizabeth H., and O'Riordan, Kate. 2002. Ethics of Internet research: Contesting the human subjects research model. Ethics and Information Technology 4(3):233-247.

Boehlefeld, Sharon Polancic. 1996. Doing the right think: Ethical cyberspace research. The Information Society 12(2):141-152.

Bruckman, Amy. 2002. Studying the amateur artist: A perspective on disguising data collected in human subjects research on the Internet. Ethics and Information Technology 4(3):217-231.

Bull, Sheana, and McFarlane, Mary. 2000. Soliciting sex on the Internet: What are the risks for sexually transmitted diseases and HIV? Sexually Transmitted Diseases 27(9):545-550.

Cavazos, Edward A., and Morin, Gavino. 1994. Cyberspace and the law: Your rights and duties in the on-line world. Cambridge, MA: MIT Press.

Danet, Brenda.2001a.Cyberpl@y: Communicating online. New York: New York University Press.

Danet, Brenda. 2001b. Studies of cyberpl@y: Ethical and methodological aspects. Case study prepared for the Ethics Working Group, Association of Internet Researchers. 〈http://atar.mscc.huji.ac.il/ msdanet/papers/ethics2.pdf $\rangle$

Department of Health, Education, and Welfare. 1979. The Belmont report: Ethical principles and guidelines for the protection of human subjects of research. Washington, DC: OPRR Reports.

Elgesem, Dag. 2002. What is special about the ethical issues in online research? Ethics and Information Technology 4(3):195203.

Ess, Charles. 2002. Ethical decision-making and Internet research: Recommendations from the AoIR Ethics Working Committee. Association of Internet Researchers (AoIR). 〈http://aior.org/reports/ ethics.pdf $\rangle$

Eysenbach, Gunther, and Till, James E. 2001. Ethical issues in qualitative research on Internet communities. British Medical Journal, 10 November:1103-1105.
Frankel, Mark S., and Siang, Sanyin. 1999. Ethical and legal aspects of human subjects research on the Internet. American Association for the Advancement of Science (AAAS). 〈http://www.aaas.org/ spp/sfri/projects/intres/report.pdf $\rangle$

Headland, Thomas N., Pike, Kenneth, and Marvin, Harris, eds. 1990. Emics and etics: The insider/outsider debate. Newbury Park, CA: Sage Press.

Herring, Susan. 1996. Linguistic and critical analysis of computermediated communication: Some ethical and scholarly considerations. The Information Society 12(2):153-168.

Herring, Susan C. 1999. The rhetorical dynamics of gender harassment online. The Information Society 15(3):151-167.

Hudson, James M., and Bruckman, Amy. 2002. IRC Français: The creation of an Internet-based SLA community. Computer Assisted Language Learning 15(2):109-134.

Kant, Immanuel. 1981. Grounding for the metaphysics of morals, trans. J. W. Ellington. Indianapolis, IN: Hackett.

King, Storm A. 1996. Researching Internet communities: Proposed ethical guidelines for reporting of results. The Information Society 12(2):119-127.

Kraut, Robert, Olson, Judith, Banaji, Mahzarin, Bruckman, Amy, Cohen, Jeffery, and Mick, Couper. 2004. Psychological research online: Report of Board of Scientific affairs' advisory group on the conduct of research on the internet. American Psychologist 59(4):1-13.

Latané, Bibb, and Darley, John M. 1970. The unresponsive bystander: Why doesn't he help?, eds. K. MacCorquodale, G. Lindzey, and K. E. Clark. Century Psychology Series. New York: Appleton-CenturyCrofts.

Milgram, Stanley. 1974. Obedience to authority: An experimental view. New York: Harper Perennial.

Mill, John Stuart. 1998. Utilitarianism. In J. S. Mill: Utilitarianism, ed. R. Crisp. New York: Oxford University Press.

Nosek, Brian A., Banaji, Mahzarin R., and Greenwald, Anthony G. 2002. E-Research: Ethics, security, design, and control in psychological research on the Internet. Journal of Social Issues 58(1):161176.

Pedhazur, Elazar J. 1997. Multiple regression in behavioral research: Explanation and prediction, 3rd ed. New York: Harcourt.

Reid, Elizabeth. 1996. Informed consent in the study of on-line communities: A reflection on the effects of computer-mediated social research. The Information Society 12(2):169-174.

Rosaldo, Renato. 1989. Culture and truth: The remaking of social analysis. Boston: Beacon Press.

Schrum, Lynne. 1997. Ethical research in the information age: Beginning the dialog. Computers in Human Behavior 13(2):117-125.

Walther, Joseph B. 2002. Research ethics in internet-enabled research: Human subjects issues and methodological myopia. Ethics and Information Technology 4(3):205-216.

Waskul, Dennis, and Douglass, Mark. 1996. Considering the electronic participant: Some polemical observations on the ethics of on-line research. The Information Society 12(2):129-139. 